\title{
Analysis and Synthesis of Electrocardiogram (ECG) using Fourier and Wavelet Transform
}

\author{
Mohammed Basheer Mohiuddin ${ }^{a}$, Isam Janajreh ${ }^{a}$ \\ ${ }^{a}$ Khalifa University, Abu Dhabi, United Arab Emirates
}

\begin{abstract}
Electrocardiogram (ECG) is the study of the electrical signals of the human heart that are generated by the pumping action of the heart caused by the polarization and depolarization of the nodes of the heart. These signals must be interpreted with great accuracy and efficiency as they are paramount in prognosis and subsequent diagnosis of the condition of the patient. The goal of this project is to analyze the ECG signals following Fourier and Wavelet transforms, and to highlight and demonstrate the advantages of the Wavelet transform. Firstly, it involves simulating the temporal digital ECG signal and explaining the signal constituents, i.e., P, Q, R, S, T waves while staying in the time domain. Secondly, the ECG signal will be transferred into the frequency domain for quick, fast, and compressed analysis and carry out signal processing using Fourier analysis and highlight the pros and cons of this technique. Thirdly, wavelet analysis will be explored and demonstrated to mitigate the shortcoming of the former tool, i.e., Fourier. At this stage, various ECG signals, mimicking abnormalities, will be analyzed. This work will highlight the effectiveness of wavelet analysis as a tool to examine ECG signals. This work, hence, will entail, comparison of both transformation methods by utilizing the computational power of MATLAB.
\end{abstract}

Keywords: ECG, Fourier transform, Hann window, Wavelet transform, Daubechies, Symlet

\section{Introduction}

Ever since the inception of ECG at the turn of the $20^{\text {th }}$ century, it has been an important tool for medical doctors to study and understand the functioning of the human heart. ECG has played an important role in helping practitioners to diagnose cardiac conditions and treat them accordingly. Currently, with an unprecedented rate of improvement of technology, ECG signals have been studied extensively. Numerous analysis and signal processing techniques have been employed for this purpose, ranging from various Fourier transform techniques like Fast Fourier Transform (FFT), zoom FFT to the more common Wavelet analysis. Apart from these methods, other techniques like Neural networks or differential equation procedures have been utilized successfully.

Fourier transform has been used for ECG signal synthesis for a long time now. The authors in [1], [2] utilized the Fourier

* Corresponding author

E-mail: 100059790@ku.ac.ae

(c) 2016 International Association for Sharing Knowledge and Sustainability

DOI: $10.5383 /$ ijtee. 17.02 .002 series technique to generate the normal and abnormal ECG signals. However, no further analysis was performed in this work. Bennet et al. [3] came up with an interesting use of Fourier analysis of ECG vis-à-vis its shortcomings. The authors came up with a device to detect only two conditions, namely, tachycardia and bradycardia. These abnormalities depend only on the heart rate, which is easily measurable through FFT. Similarly, Lukáč and Ondráček [4] took advantage of this use of FFT and used it to calculate the heart rate.

Parak and Havlik [5] used statistical and differential mathematical tools to de-noise the ECG signal before utilizing it for making an implementable method for a real-time stress test. The proposed algorithm could work even in the presence of disturbance from the movement of muscles. Their main goal was to design a digital computing algorithm that could be implemented in real-time. Hence, the differential approach was very fast and effective. Murugan and Ramesh [6] used the zoom FFT as a less explored technique for analyzing ECG signals. They produced the ECG waveform using MATLAB code and then used the zoom FFT technique to detect the QRS complex and $\mathrm{P}$ and $\mathrm{T}$ peaks. The obtained results were compared with 
those obtained by conventional FFT practices. It concluded that the quality of spectrum for ECG analysis was better when using zoom FFT which in turn was better for diagnosing cardiac conditions and all this was obtained while not saturating the processor capabilities. In [7]-[29] the authors used Wavelet Transform techniques to analyze ECG signals. As a first step, the authors filtered the noise in ECG signals which were traced to various sources such as from the electric wires involved, and muscle activity. The use of the Daubechies family [30] of the wavelet transform was abundantly found in the literature. In [10], the author used $d b 2$ of Daubechies family of Continuous Wavelet Transform (CWT) as it is suggested to provided better diagnostic ability. In [11], the author utilized $d b 6$ of the Daubechies family of orthogonal wavelets, whereas in [13] the authors used $d b 10$. In all these works, the efficiency of using wavelets was highlighted. Castro et al. used an optimal mother wavelet technique in their work [31]. Rather than using a predefined wavelet family, the authors found out the wavelet that fits a specific ECG signal. Tamil et al. [32] used the Discrete Wavelet Transform (DWT), also discussed in [14], [18], [22], [27], for extraction of the characteristics of the ECG signal which was then fed to a hybrid neuro-fuzzy system consisting of Neural Networks and Fuzzy Logic. This method proved to be very accurate. However, due to the lack of an adequate database for various heart ailments, there is still room for improvement. The diagnostic ability, though, was increased considerably by using this hybrid system. Largely all the work harnessing the benefits of wavelet transform utilizes the coefficients, conversely, Peng and Wang [21] took a different approach where they employed the eigenvalues for detecting myocardial abnormalities [33] in the human through the recorded ECG signals. Daamouche et al. [23] classified the ECG signals using a polyphase representation of wavelet filter bank through a particle swarm optimization framework. The authors concluded that the proposed method was more effective than using standard wavelets like Daubechies and Symlet at the cost of far higher computational time.

The use of wavelet was not only limited to diagnosis rather was utilized even for matching the shape of a wavelet with the ECG signal [34]. Apart from the use of CWT and DWT, Cross Wavelet Transform was explored in the literature too [35]-[38] as well as their intermittency factor and energy percentage contribution within the signal [39]. However, the accuracy was not as high in this case as compared to the conventional wavelet techniques.

Most of the work focused on detecting heart diseases and cardiac conditions from the analyzed ECG signals, however, Sasikala and Wahidabanu [12], Mahmoud and Jusak [40] and Dar et al. [41] took the work further. They not only analyzed the ECG signal but also attempted to find a novel application of this analysis. They claimed that ECG signals, like fingerprints and retinal signatures, are unique to each individual and can be used as an identification tool. They presented analysis procedures to get this identification utility from these signals using Wavelet transform.

In this work, we aim to deconstruct an ECG signal using Fourier transform and a variety of orthogonal families of the Wavelet transform. These deconstructed waves will then be analyzed by time-shifting and stretching in the time domain. Following which we target to reconstruct the ECG signal using the obtained coefficients. This analysis will pave way for the synthesis of artificial heart signals and prognosis, the utility of which cannot be stressed enough in the modern day. All the work in the literature deals exclusively with only one of the two transforms.
In this paper, we aim to provide a comparative study between Fourier and Wavelet transform and highlight the effectiveness of Wavelet Transform for ECG signal investigation.

\section{ECG Signal}

The ECG signal helps us study the condition of the human heart. It has certain characteristic features that give it meaning and helps medical practitioners understand the physiology of the patient's heart. It has proved to be a life-saving tool by aiding the diagnosis and prognosis of various heart ailments.

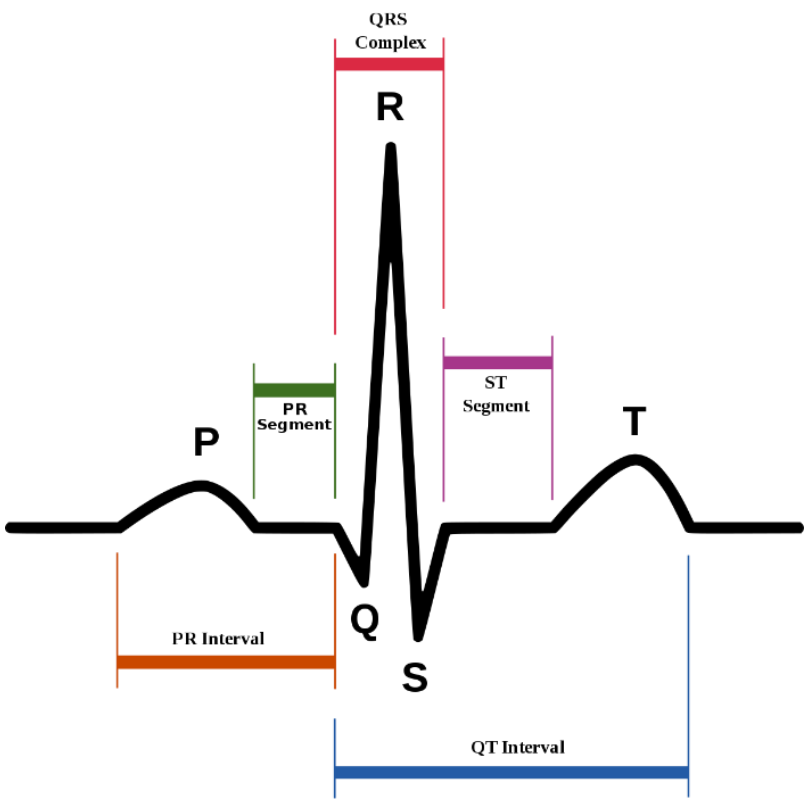

Fig. 1. Normal Sinus Rhythm

An electrocardiogram, as shown in Fig. 1 for a normal heartbeat, is composed of several 'waves' and 'segments' that are connected by an isoelectric line. Each wave and segment signify a particular action of the heart.

The first component is the 'P-wave' which indicates the depolarization of the sinoatrial node. This wave has a typical duration of $100 \mathrm{~ms}$ and a peak value of $0.3 \mathrm{mV}$. The most distinguishing trait of the ECG signal is the 'QRS complex'. This complex is made up of 3 waves, Q-, R-, and S- waves. The $\mathrm{Q}$-wave and $\mathrm{S}$-wave are negative parts and $\mathrm{R}$-wave is the highest peak in the ECG signal. The 'QRS complex' has a time duration between 50 to $110 \mathrm{~ms}$. The final wave is the 'T-wave' which shows the process of repolarization of the heart. The heart returns to its idle state during this wave. This 'T-wave' has an amplitude of around $0.8 \mathrm{mV}$ and lasts around $0.42 \mathrm{~ms}$.

Apart from the waves, another important part of the ECG signal is the connecting intervals. These segments are isoelectric components, i.e., the voltage remains at $0 \mathrm{mV}$ during these intervals. The 2 intervals of interest are 'P-R interval' and 'S-T interval'.

The ECG signals used in this work are obtained from the PhysioNet master database [42] which contains modified copies of 3 PhysioNet databases [42]-[44]. This contains pre-filtered Normal Sinus Rhythm, various arrhythmia signals, and congestive heart failure records. For analysis, only the initial 10 seconds of the data is considered. Since the signal is sampled at $128 \mathrm{~Hz}$, it gives enough data points (1280) in the span of 10 seconds to effectively evaluate the signal without much 
computational burden. The signal segment used is shown in Fig 2 . The peak detection is a very important phenomena related to ECG signal. It gives us a measure of the heart rate which is the basis of many pathological condition detection. The peak detection includes primarily includes detecting the ' $\mathrm{R}$-wave'. The time interval between 2 consecutive 'R-waves' helps us calculate heart rate of the patient.

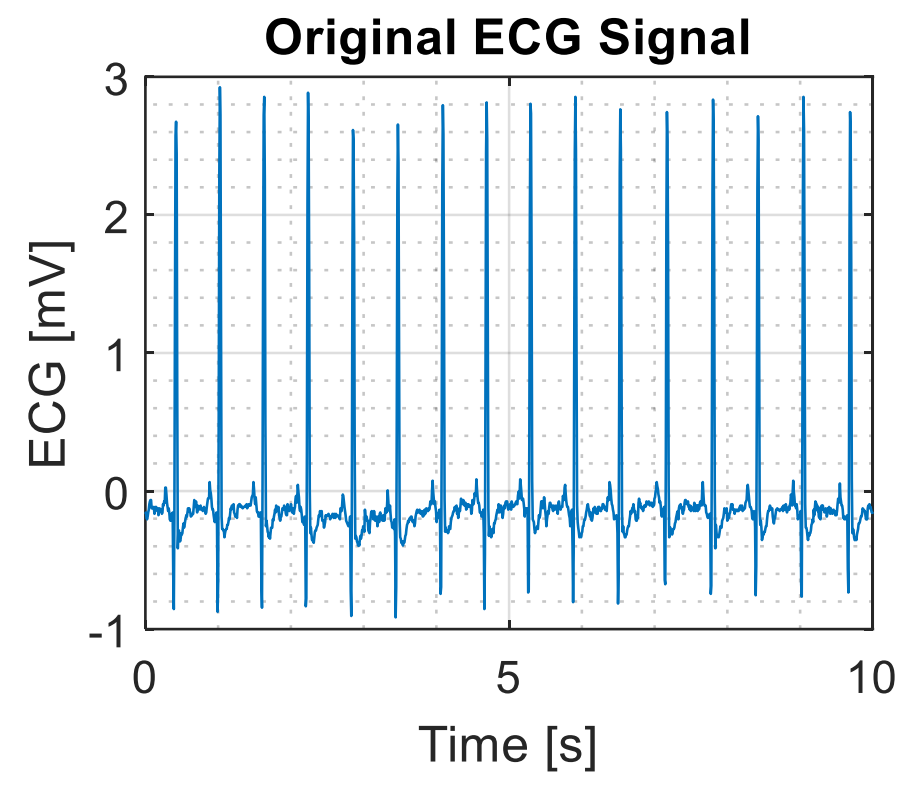

Fig. 2 Filtered ECG signal segment

The heart rate of the sample shown in Fig. 1 was 95 beats per minute (bpm), and the value obtained through the peak detecting algorithm was $96 \mathrm{bpm}$. Furthermore, peak detection also includes detecting all the waves in the ECG signal. In Fig. 3 we see that all the waves are characterized by their crests and troughs.

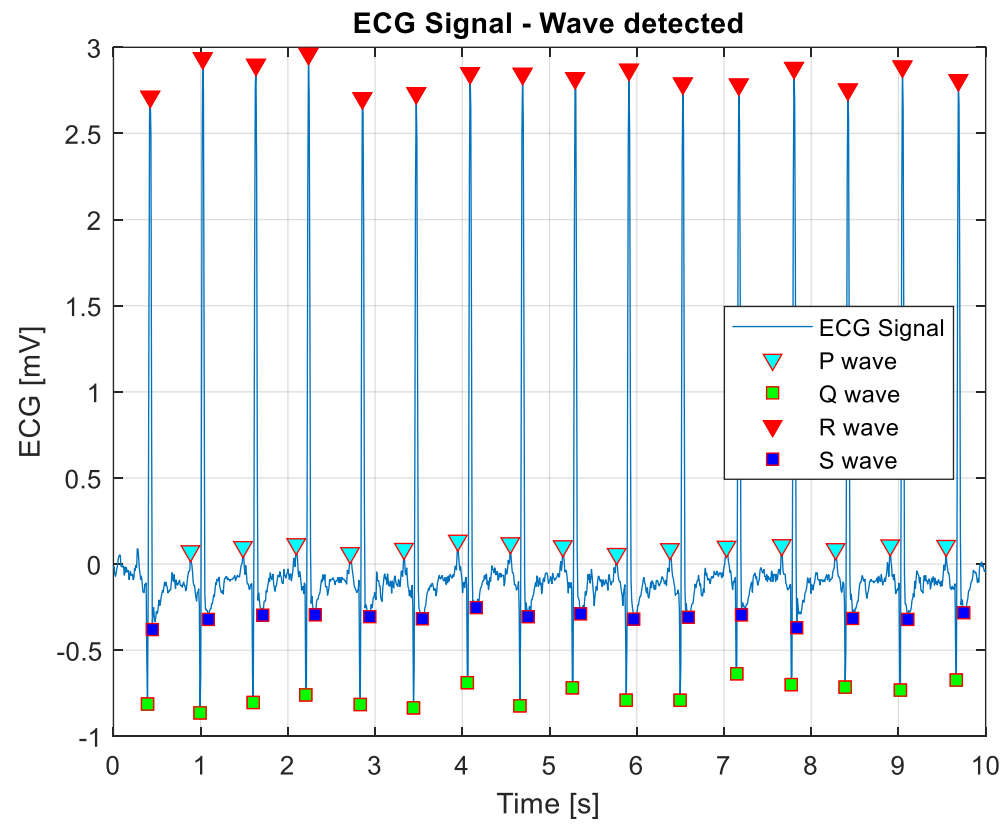

Fig. 3 ECG signal peak and wave detection

\section{Fourier Transform}

In this section, we will be discussing the use of Fourier transform for ECG analysis. Fourier transform is a powerful tool for analyzing stationary signals. The frequency-domain analysis gives a lot of information about the signal. However, when nonstationary signals, like ECG, are to be analyzed, Fourier transform falls short. Small changes in the heart rhythm most likely will go undetected if analyzed through Fourier transform. We perform Fast Fourier Transform (FFT) on the ECG signal. This gives us information about the High Frequency (HF) and 
Low Frequency (LF) components of the ECG. The LF gives information about the physiological activities of the heart whereas HF indicates respiratory activity.

To analyze the signal using Fourier Transform, we perform FFT on the ECG signal. This gives us the frequency domain response of the signal.

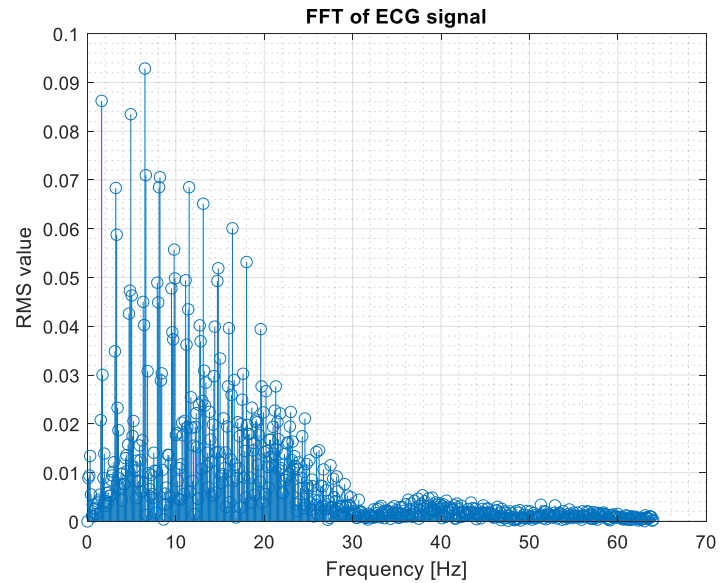

Fig. 4 FFT of ECG signal

We see in Fig. 4 that many frequency components are needed to characterize an ECG signal. This becomes clear when we plot an envelope curve of the peaks of FFT response.

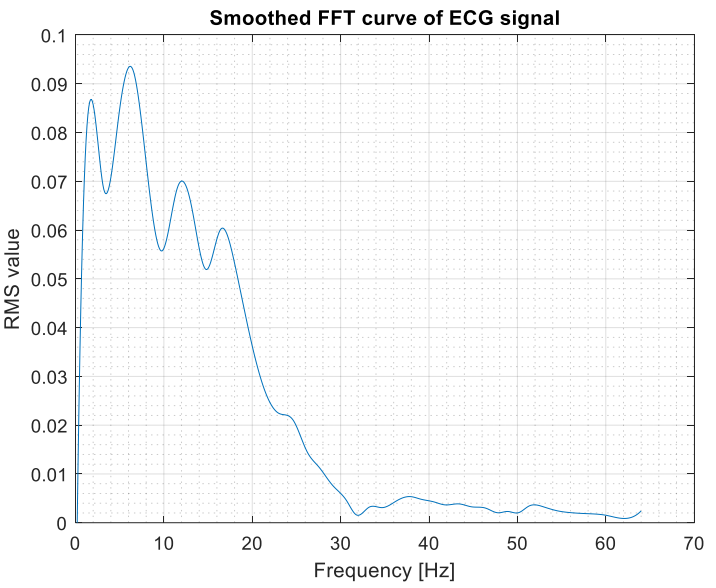

Fig. 5 Peak envelope of FFT

The response shown in Fig. 5 corresponds to a broadband response, re-iterating the point that several data points are needed to recreate the ECG signal using Fourier Transform.

FFT often falls short in accurately extracting data from nonstationary data and is not a very efficient approach. To examine this issue further, we tried to use the Hann smoothing method using a moving average window. In this method, we created 'Hann' windows of $1 s$ time duration with overlap to cover the entire sample signal length as shown in Fig. 6.
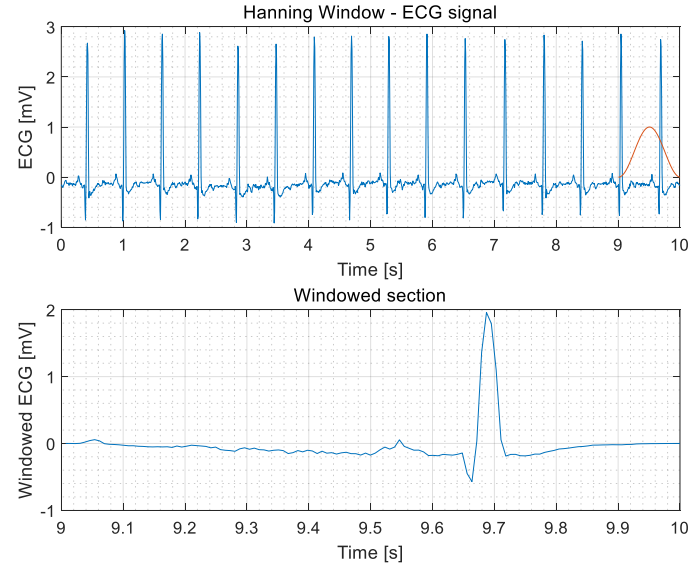

Fig. 6 Hanning of ECG signal

Single-sided FFT was performed on this windowed signal and an average was taken to investigate the frequency response of the ECG signal depicted in Fig. 7. It can be seen that despite taking an average of 19 windows, the ECG frequency response is broadband. This implies that it is not useful to analyze an ECG signal using FFT.

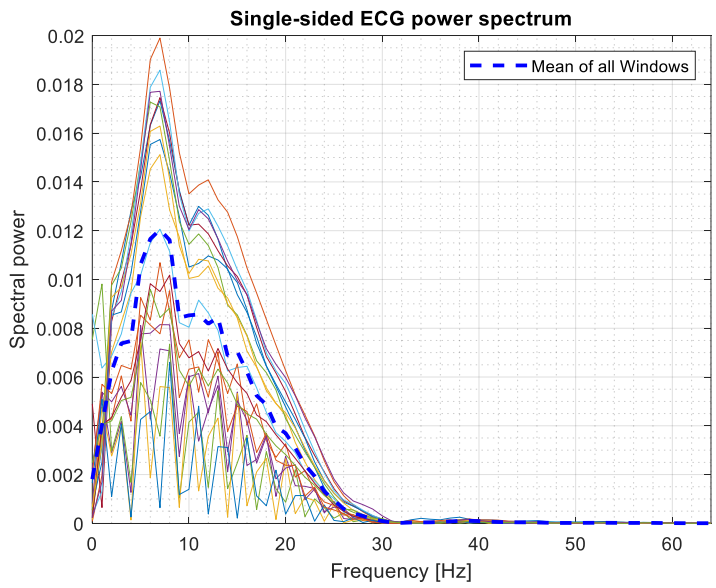

Fig. 7 ECG Power Spectrum for various Hann windows

This idea is supported by trying to recreate ECG for normal sinus rhythm using an 8-term Fourier series. The signal synthesized by Fourier series is compared with the original ECG signal in Fig. 8.

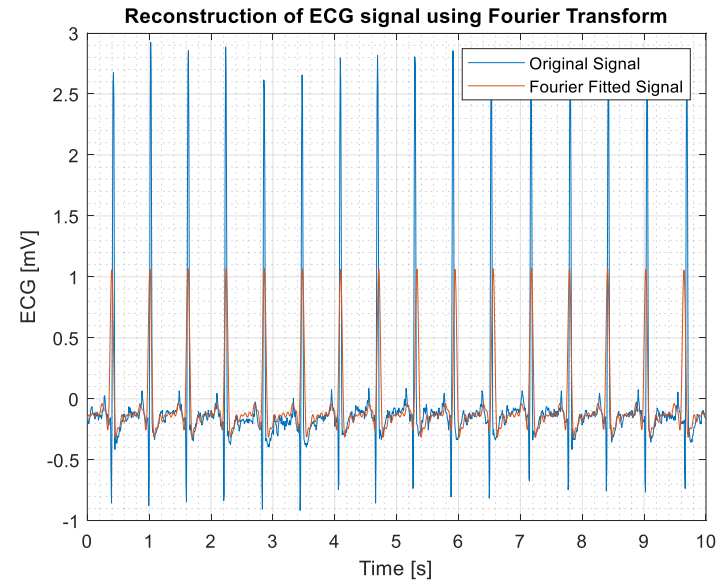

Fig. 8 Fourier transform reconstruction 
The Fourier model used to generate the Fourier series is given in Eq. (1). The corresponding coefficient values are given in Table 1 which are within a $95 \%$ confidence bound.

$$
\begin{aligned}
f(x)=a_{0}+a_{1} & \cos (x w)+b_{1} \sin (x w) \\
& +a_{2} \cos (2 x w)+b_{2} \sin (2 x w) \\
& +a_{3} \cos (3 x w)+b_{3} \sin (3 x w) \\
& +a_{4} \cos (4 x w)+b_{4} \sin (4 x w) \\
& +a_{5} \cos (5 x w)+b_{5} \sin (5 x w) \\
& +a_{6} \cos (6 x w)+b_{6} \sin (6 x w) \\
& +a_{7} \cos (7 x w)+b_{7} \sin (7 x w) \\
& +a_{8} \cos (8 x w)+b_{8} \sin (8 x w)
\end{aligned}
$$

Table 1 Fourier series coefficients

\begin{tabular}{|c|c|c|c|}
\hline Coefficients & Value & Coefficients & Value \\
\hline$a_{0}$ & -0.4492 & $w$ & 10.19 \\
\hline$a_{1}$ & -0.1526 & $b_{1}$ & -0.1114 \\
\hline$a_{2}$ & -0.02265 & $b_{2}$ & 0.1919 \\
\hline$a_{3}$ & 0.1812 & $b_{3}$ & -0.08022 \\
\hline$a_{4}$ & 0.1707 & $b_{4}$ & -0.08524 \\
\hline$a_{5}$ & 0.03242 & $b_{5}$ & 0.1387 \\
\hline$a_{6}$ & 0.05021 & $b_{6}$ & -0.07768 \\
\hline$a_{7}$ & -0.06914 & $b_{7}$ & 0.00465 \\
\hline$a_{8}$ & 0.03006 & $b_{8}$ & 0.03388 \\
\hline
\end{tabular}

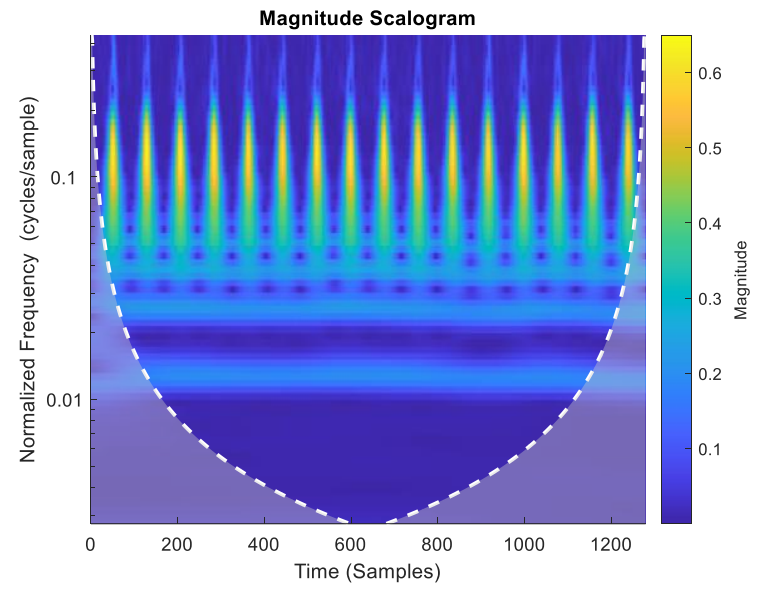

Fig. 9 CWT response a) Normal Sinus Rhythm b) Congestive heart failure

\subsection{Discrete Wavelet Transform}

In practice, when computers are used for implementing CWT then it must be in a discrete form giving rise to DWT. Being continuous causes redundancy in CWT. This problem is ably addressed by sampling CWT function in a dyadic grid. Hence, DWT is obtained by convoluting the signal with the orthonormal
It is evident that the Fourier series representation fails to replicate the peaks of the ECG signal. Due to this, important information maybe lost and hence, Fourier transform falls short in analyzing ECG signals.

\section{Wavelet Transform}

In this section, we will utilize Wavelet transform to analyze ECG signals. Subsequently, we will discuss its application in clinical prognosis. Wavelet transform is a potent means for analyzing non-stationary signals. The most important feature of the Wavelet transform is that it retains the time-domain information of the signal while also enabling the analysis in frequencydomain. This is paramount in the assessment of non-stationary waves.

Wavelet transform is of two types, Continuous Wavelet Transform (CWT) and Discrete Wavelet Transform (DWT). Both these transforms can be used effectively for diagnosis using ECG signals.

\subsection{Continuous Wavelet Transform}

The CWT of a function $f(t)$ is obtained by the following equation:

$$
W_{c}(b, a)=|a|^{\frac{1}{2}} \int_{-\infty}^{\infty} f(t) \psi^{*}\left(\frac{t-b}{a}\right) d t
$$

Where, $a, b \in R, a \neq 0$ are the scaling and shifting coefficients of the mother wavelet denoted by $\psi(t)$ respectively. The mean of a wavelet signal is zero, implying that the net area of the mother wavelet is zero.

CWT gives the spectrogram of the ECG signal. This helps us understand the signal effortlessly. It clearly shows the difference between normal and abnormal heart activity. It is seen in Fig. 9 the obvious difference between the two ECG signals. It gives medical practitioners a distinct image to quickly analyze the problem of the patient.

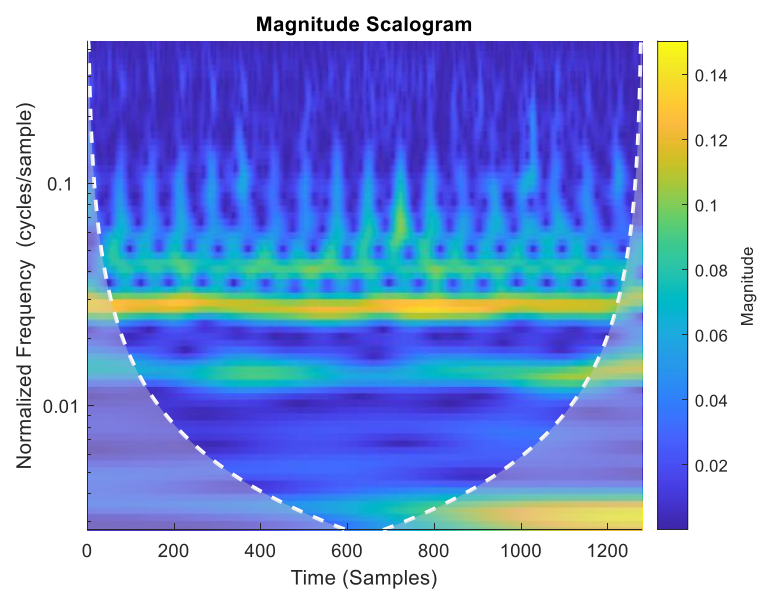

dyadic wavelet function and the scaling function. The dyadic grid is [45]:

$$
a=2^{-m} \text { and } b=n 2^{-m}
$$

where $m, n \in Z$.

From (2) and (3) we obtain the DWT function as:

$$
W_{d}(m, n)=\int_{-\infty}^{\infty} f(t) \psi_{m, n}^{*}(t) d t
$$


As an orthonormal wavelet basis is used there is no redundancy. Furthermore, we obtain a Multi-Resolution Analysis (MRA) system, which decomposes the original ECG signal into scales of different frequency and time resolution. The fundamental concept involved in MRA is to find the average features and the details of the signal via scalar products with scaling signals and wavelets. Using these techniques, the ECG is disintegrated using an optimal mother wavelet, the wavelet that most closely resembles the shape of the original signal. The decomposition includes separating the signal into high frequency and low-frequency components. This is done by decomposing the ECG signals in many levels of approximate and detailed coefficients. The detailed coefficients are obtained from the high-frequency component of the wavelet function. The approximate coefficients, given in Eq. (5) at scale $m$ and location $n$, have the details of the scaling functions $(\phi(t))$ and are low frequency components of the signal. These approximate coefficients are further broken down based on the number of levels the signal is to be decomposed into. When the ECG signal is broken into approximate and detailed coefficients based on the frequency, it still retains the time-domain information. This not only helps us to understand what the abnormalities in the ECG signal are but also helps us find the exact point at which activity of the heart happens. This decomposed signal can then be reconstructed using the obtained coefficients without much loss of information. The wavelet decomposition and the reconstruction follow the steps as depicted in Fig. 10.

$$
S_{m, n}=\int_{-\infty}^{\infty} f(t) \phi_{m, n}(t) d t
$$

The discrete approximation of the original signal is given by:

$$
f_{0}(t)=f_{M}(t)+\sum_{m=1}^{M} d_{m}(t)
$$

Where $f_{M}$ is the mean signal approximation at scale $M$ given by $f_{M}(t)=S_{M, n} \phi_{M, n}(t)$ and $d_{M}$ is the detail signal approximation at scale $m$, given by $d_{m}(t)=\sum_{n=0}^{M-m} T_{m, n} \psi_{m, n}(t)$.

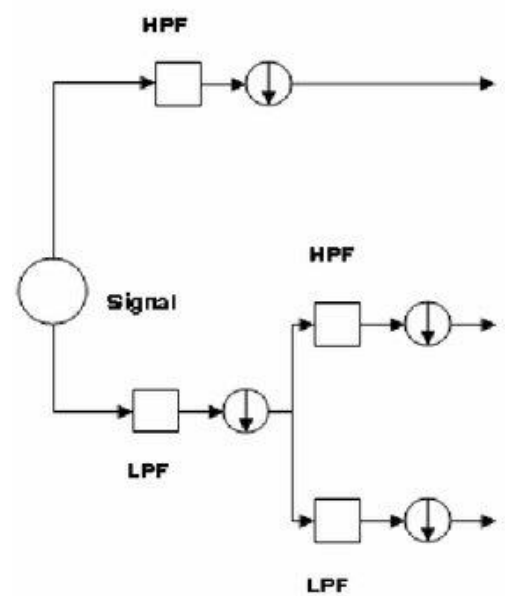

Wavelet Decomposition

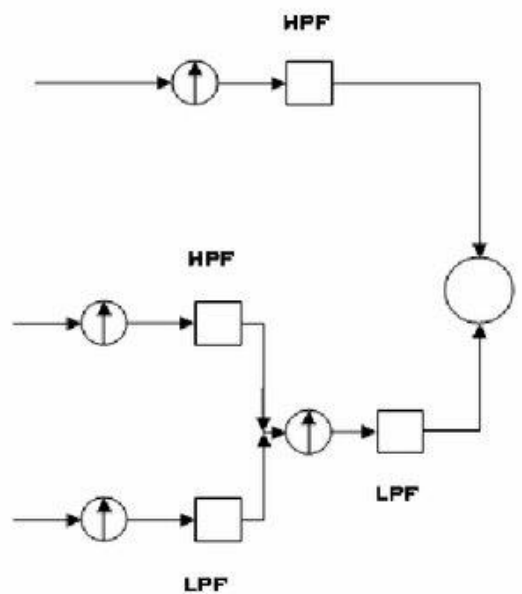

Wavelet Rescorstruction

Fig. 10 Two-level Wavelet decomposition and reconstruction [46]

From Fig. 10 we see that the approximation of the signal at a given scale is the combination of the approximate and detail at the next smaller scale given in Eq.

$$
f_{m}(t)=f_{m-1}(t)-d_{m}(t)
$$

In this work, we tried various orthogonal wavelets and decided to explore the use of Symlet wavelet, a modified version of the more popular Daubechies wavelet. This wavelet was chosen as sym 4 of the Symlet family had a shape very similar to the original normal ECG signal as shown in Fig. 11. We generated the ECG signal recreated using the aforementioned wavelet and compared it with the original ECG signal to gauge the effectiveness of the wavelet as an analysis tool for ECG signals. The plot in Fig. 12 shows that the reconstructed signal traces the normal ECG signal with great accuracy. This establishes the effectiveness of Wavelet Transform in analyzing and synthesizing ECG signals.

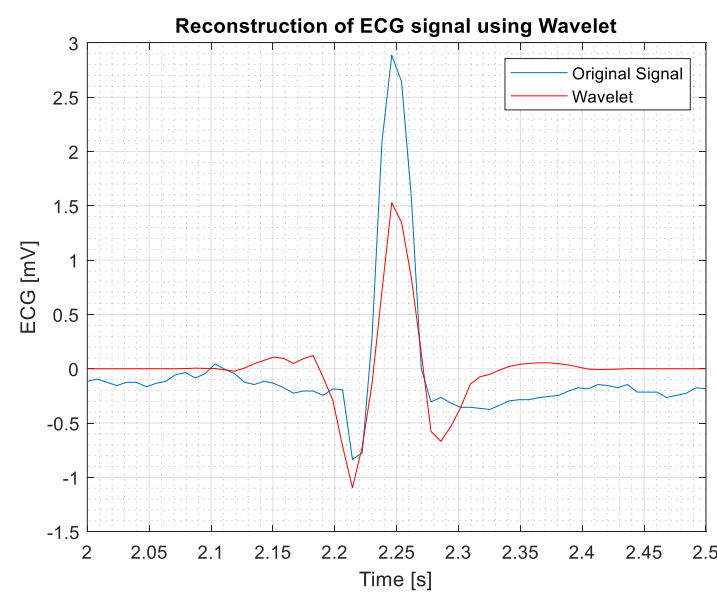

Fig. 11 Matching wavelet with ECG signal 


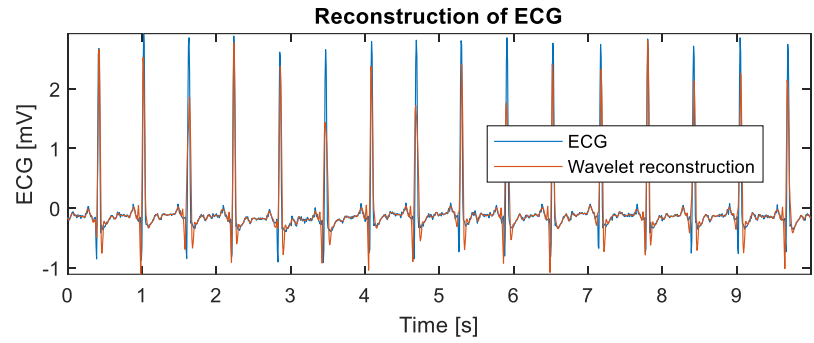

Fig. 12 Wavelet reconstruction of normal ECG signal

\subsection{Wavelet Analysis}

Wavelet analysis gives the details about the ECG signal in both the time and frequency domain. Also, wavelet decomposition gives different coefficients for different signals, implying, for all different arrhythmias and congestive heart failures, the coefficients remain distinct. This plays a major role in distinguishing the ECG and consequently in diagnosing the patient.
In this paper, we compare the decomposition coefficients are the 2 levels of the normal sinus rhythm with arrhythmias and with congestive heart failure records. Based on the comparison we will be able to classify the ECG records as arrhythmias or heart failure. This should help in a quick analysis of the patient's condition. From the plots in Fig. 13 to Fig. 22, we see that the coefficients of the normal sinus rhythm are significantly different when compared to various diseases. In each of the following figures, the normal sinus rhythm's coefficients, both decomposition, and reconstruction are compared with that of several abnormalities. In Fig. 13 and Fig. 14 the comparison gives is for hyperkalemia where the 'P-wave' is missing, and the 'T-wave' has a high magnitude. Furthermore, we compared the myocardial ischemia shown in Fig. 15 and Fig. 16, which has an inverted 'T-wave', with a normal heartbeat. Two very common arrhythmias are bradycardia, depicted in Fig. 17 and Fig. 18, where the heart rate drops below $50 \mathrm{bpm}$, and tachycardia, exhibited in Fig. 19 and Fig. 20 a case in which the heart rate exceeds 120 bpm. In Fig. 21 and Fig. 22, we see the case of congestive heart failure.
Deconstruction

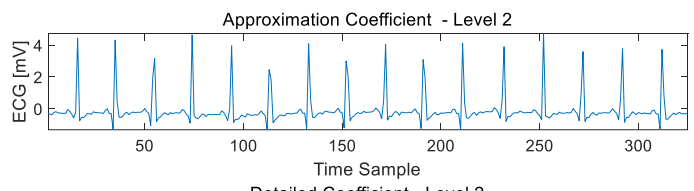

Detailed Coefficient - Level 2

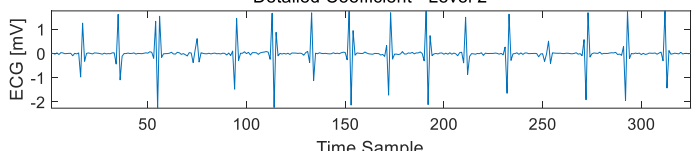

Detailed Coefficient - Level 1

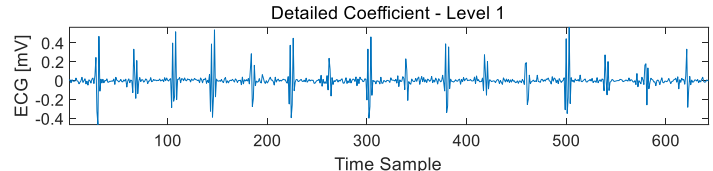

Deconstruction

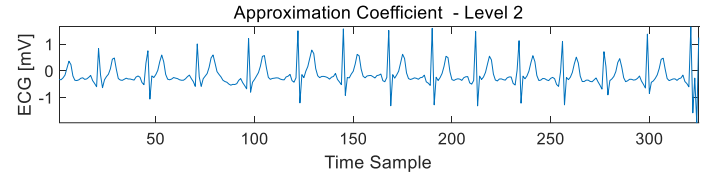

Detailed Coefficient - Level 2

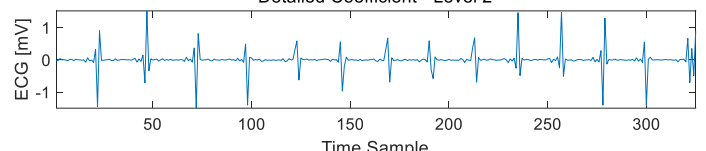

Detailed Coefficient - Level 1

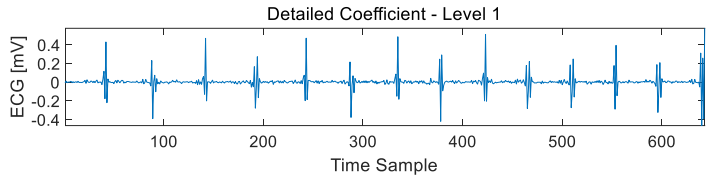

Fig. 13 Deconstruction coefficients a) Normal Sinus Rhythm b) Hyperkalemia
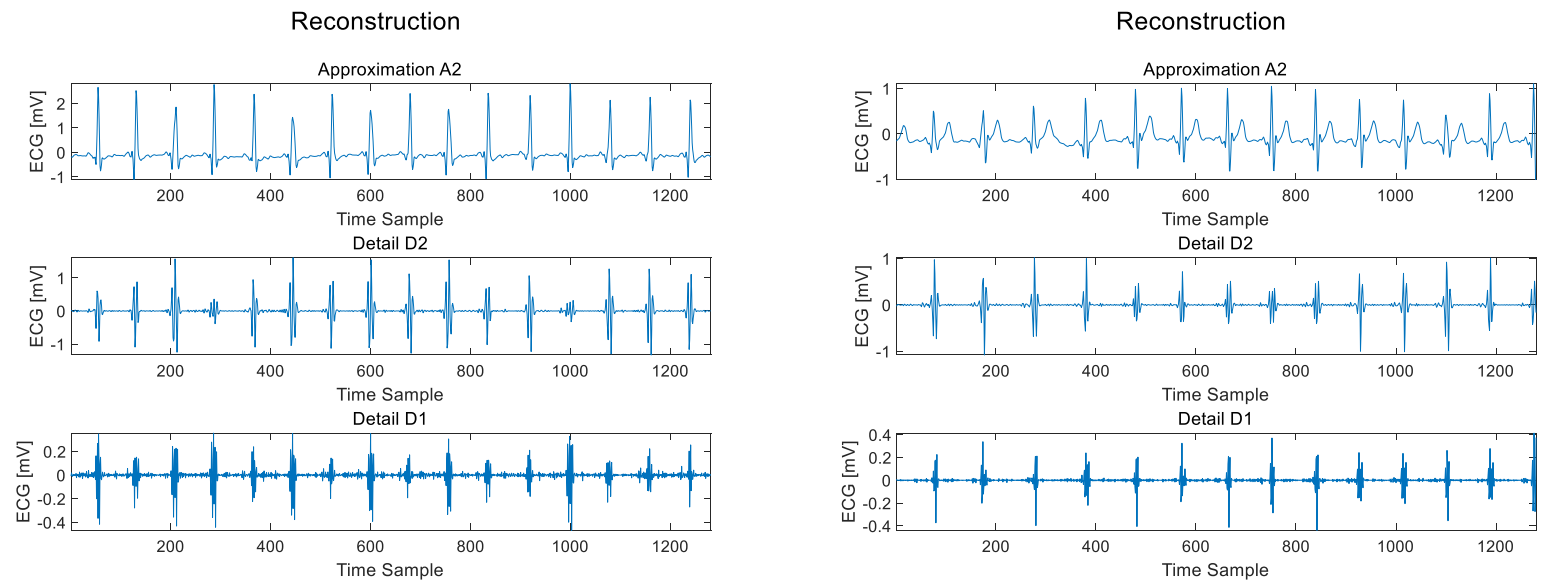

Fig. 14 Reconstruction coefficients a) Normal Sinus Rhythm b) Hyperkalemia 
Deconstruction

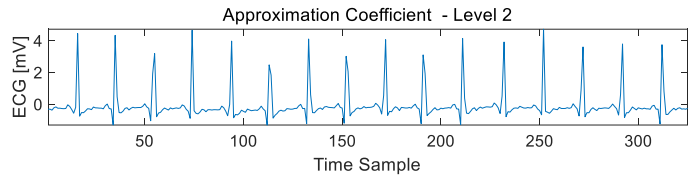

Detailed Coefficient - Level 2

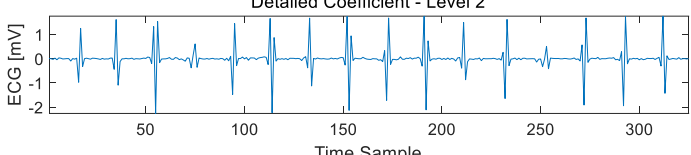

Detailed Coefficient - Level 1

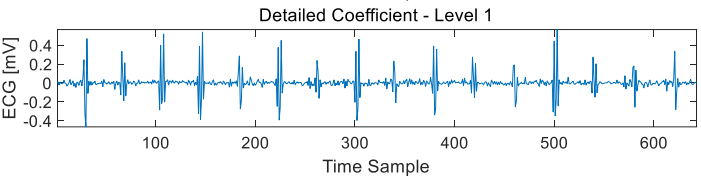

Deconstruction

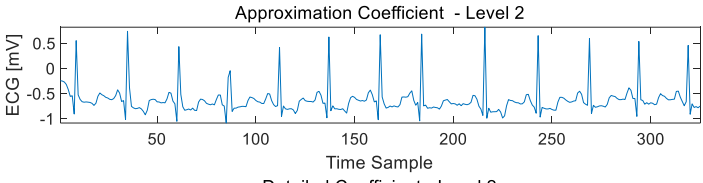

Detailed Coefficient - Level 2
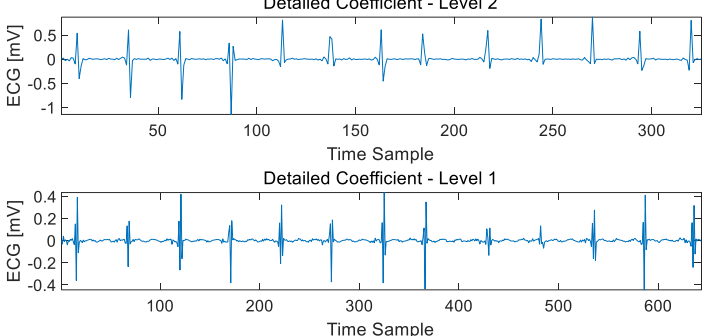

Fig. 15 Deconstruction coefficients a) Normal Sinus Rhythm b) Myocardial Ischemia
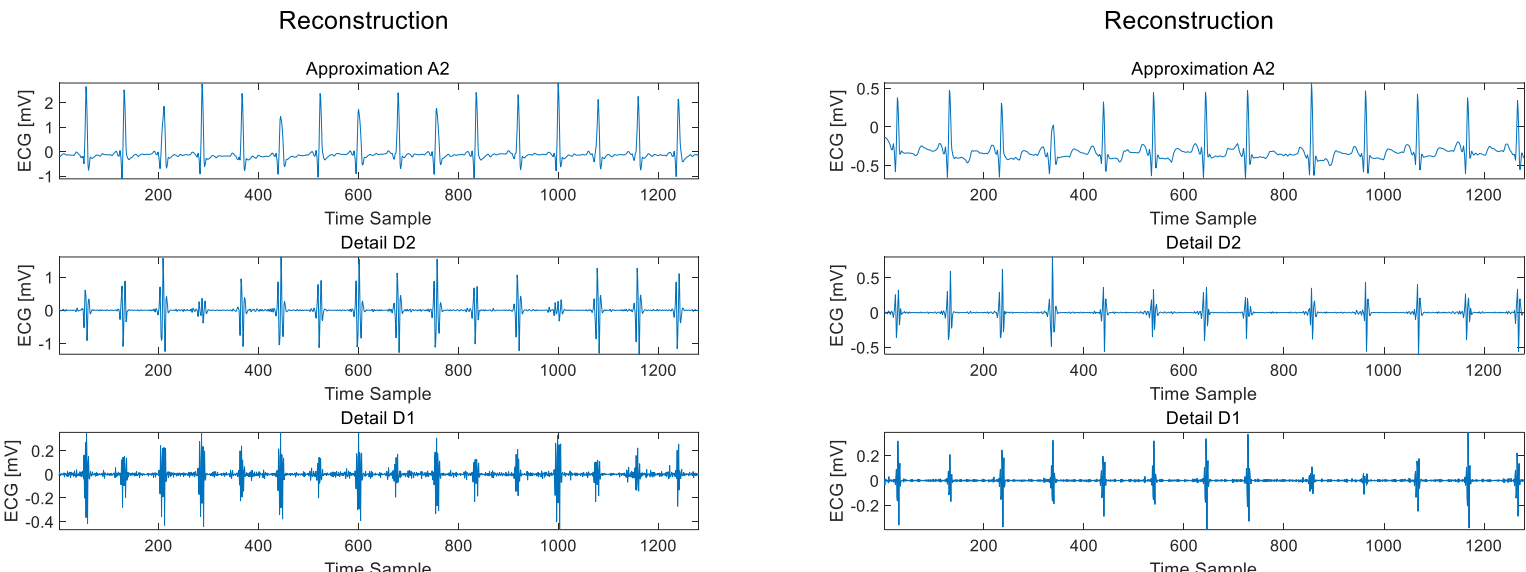

Fig. 16 Reconstruction coefficients a) Normal Sinus Rhythm b) Myocardial Ischemia

Deconstruction
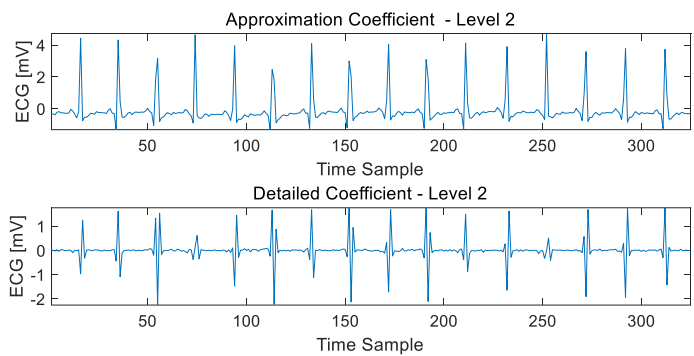

Detailed Coefficient - Level 1

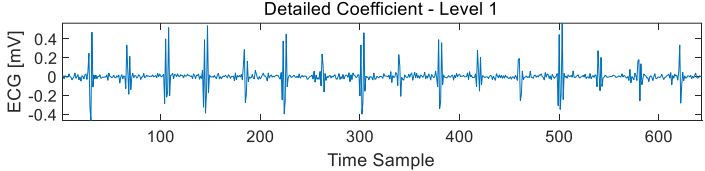

Deconstruction

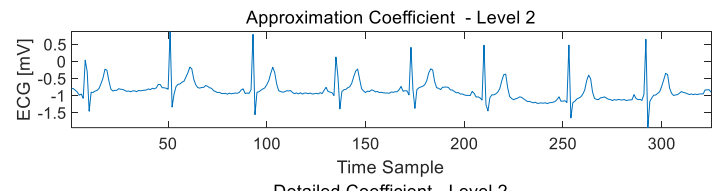

Detailed Coefficient - Level 2

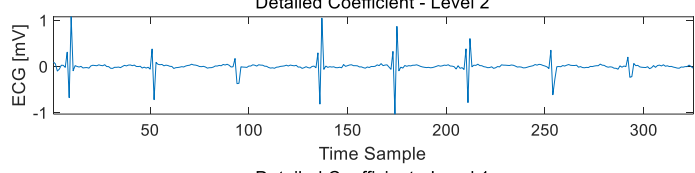

Detailed Coefficient - Level

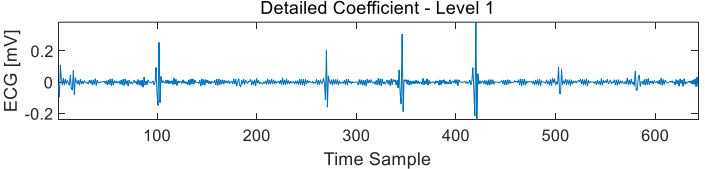

Fig. 17 Deconstruction coefficients a) Normal Sinus Rhythm b) Bradycardia 

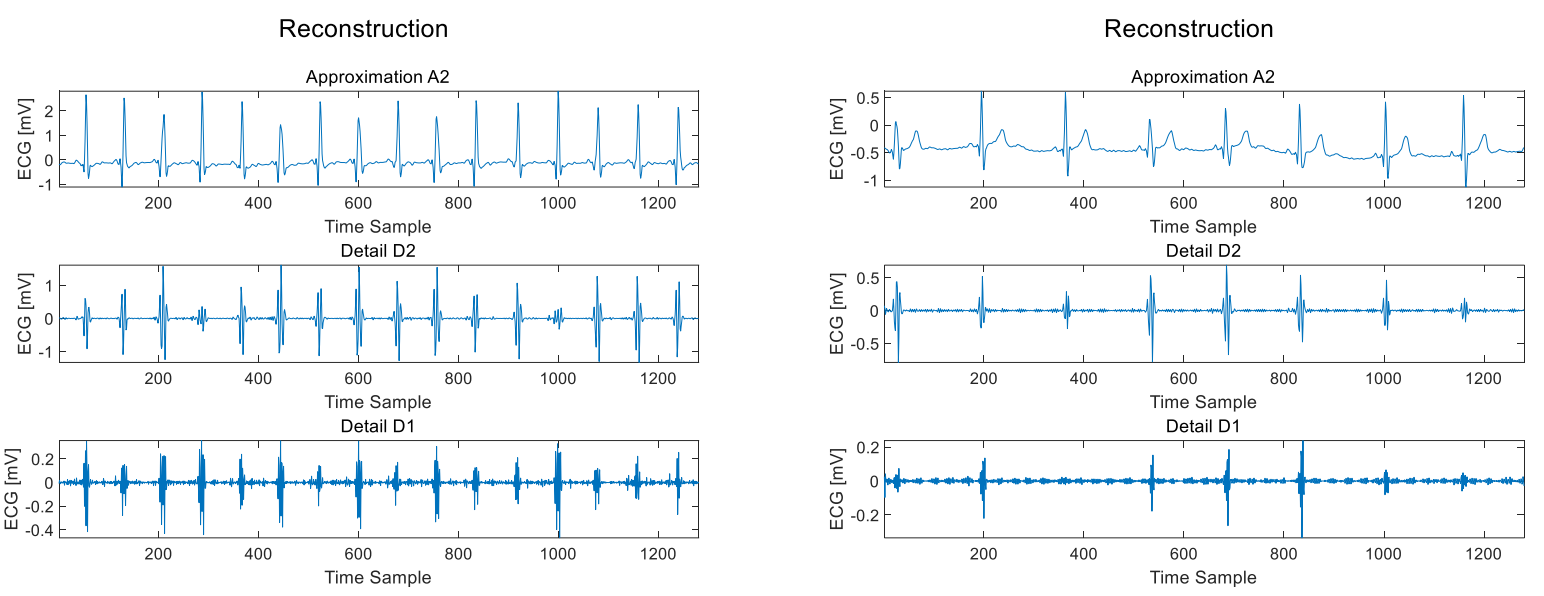

Fig. 18 Reconstruction coefficients a) Normal Sinus Rhythm b) Bradycardia

Deconstruction
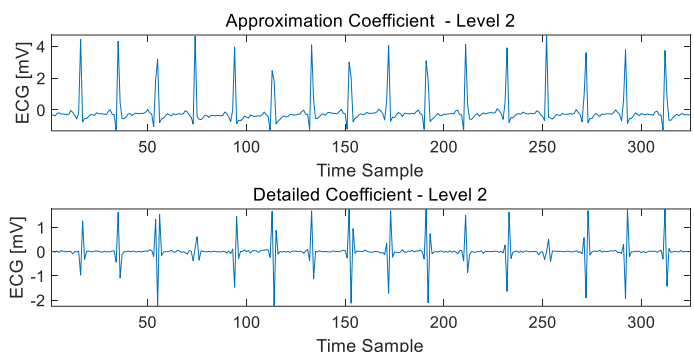

Detailed Coefficient - Level 1

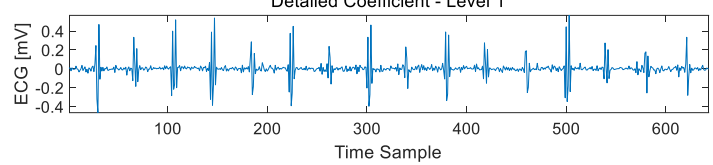

Deconstruction

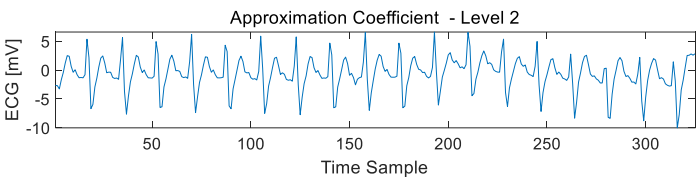
Detailed Coefficient - Level 2

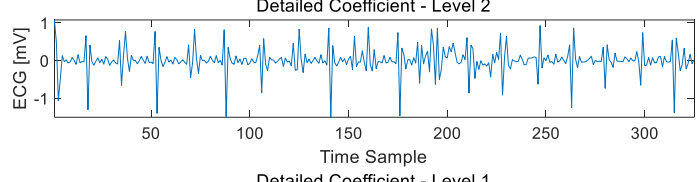

Detailed Coefficient - Level 1

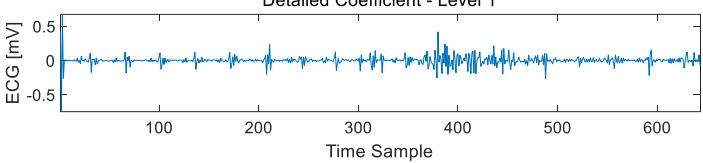

Fig. 19 Deconstruction coefficients a) Normal Sinus Rhythm b) Tachycardia
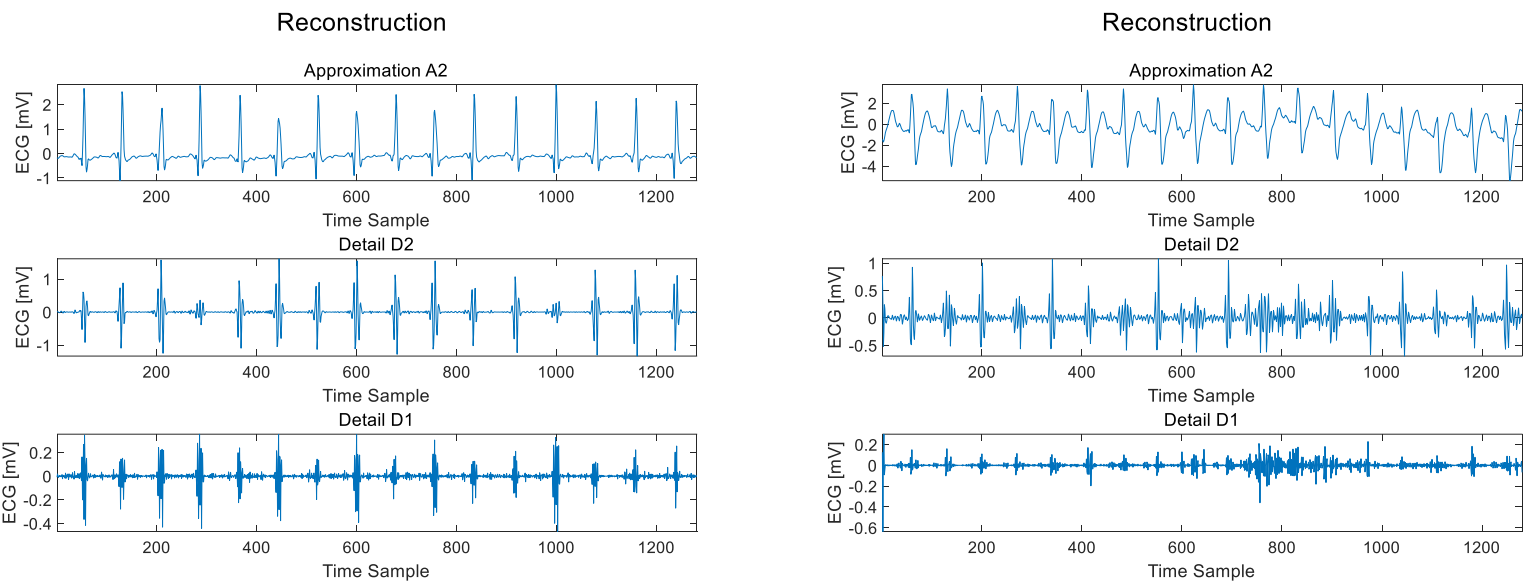

Fig. 20 Reconstruction coefficients a) Normal Sinus Rhythm b) Tachycardia 
Deconstruction

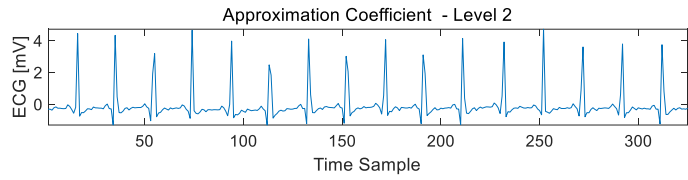

Detailed Coefficient - Level 2

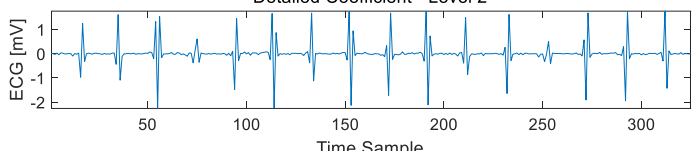

Detailed Coefficient - Level 1

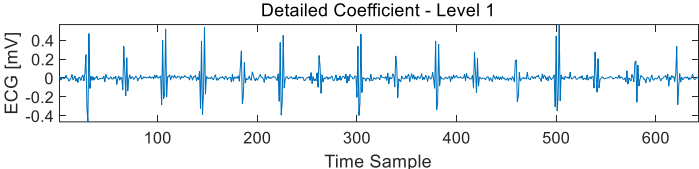

Deconstruction

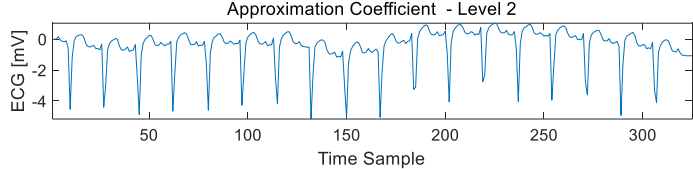

Detailed Coefficient - Level 2

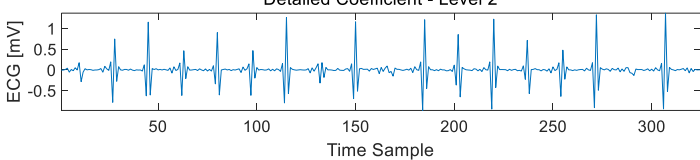

Detailed Coefficient - Level 1

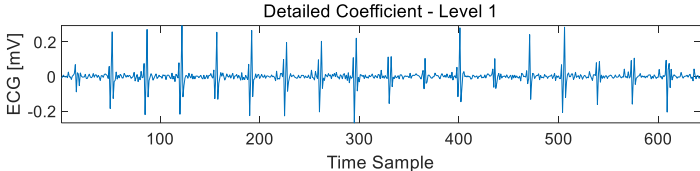

Fig. 21 Deconstruction coefficients a) Normal Sinus Rhythm b) Congestive heart failure
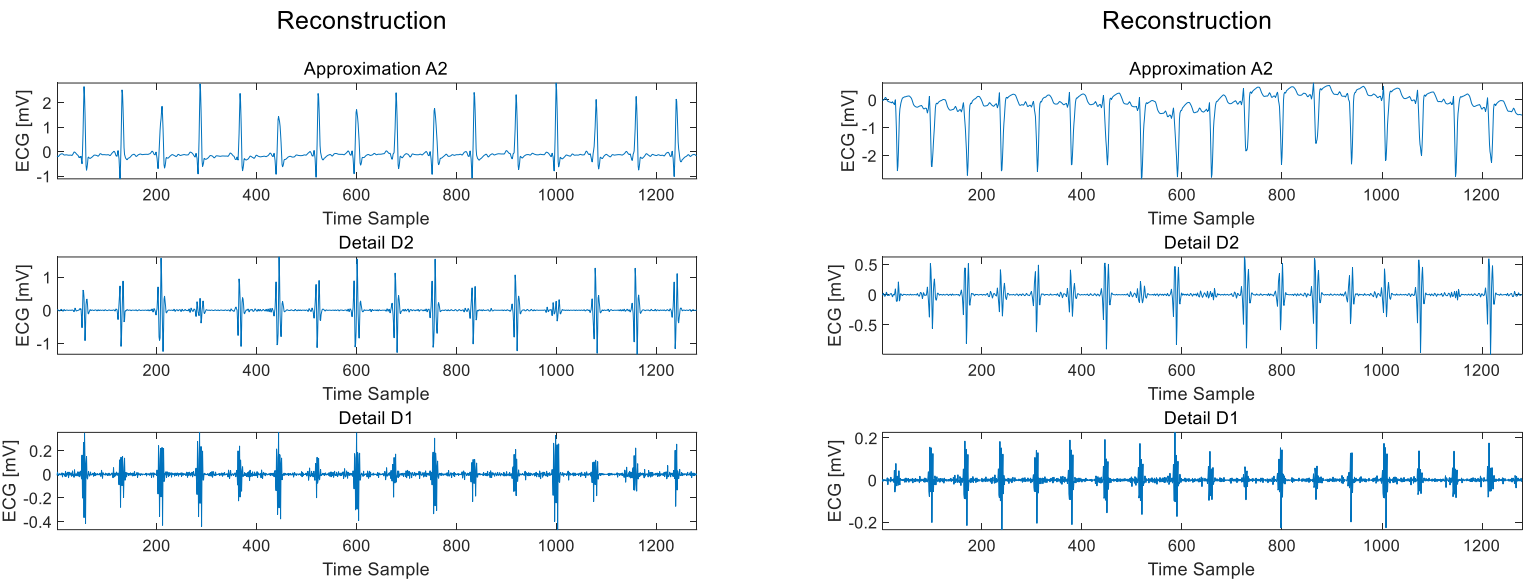

Fig. 22 Reconstruction coefficients a) Normal Sinus Rhythm b) Congestive heart failure

\section{Conclusion}

To begin with, we studied the effectiveness of the Fourier Transform in analyzing ECG signals. It was seen that it was not very effective and could not give full information on the ECG signal because it analyzes a signal only in the frequency-domain. In that way, the time localization information is lost. Hann window technique was deployed too for extracting more information using FFT. However, even this technique failed as was seen when we tried to reconstruct the ECG wave using the coefficients obtained from the aforementioned tools.

This work was then advanced to study ECG signals using Wavelet Transform. Wavelet has been an efficient tool for analyzing non-stationary signals like ECG. Firstly, we used the CWT method for analyzing the heartbeat signals. It was seen that this provided an uncomplicated way to understand the signal by giving a spectrographic representation of the signal, in which any abnormalities were pronounced. Additionally, we investigated the use of DWT for this purpose. We saw that this was very effective as it addressed the shortcomings in Fourier analysis effectively. This was primarily due to the ability of wavelet analysis to analyze a signal in both time- and frequencydomain. This helped us understand the exact abnormality at the exact instant in time. This is paramount in diagnosis. From this, we can conclude that wavelet transform was superior to Fourier in terms of examining the ECG signal. This implies that Wavelet Transform can be an effective clinical tool to analyze ECG signals and accurately diagnose heart conditions.

\section{Nomenclature}

$a_{n} \quad$ Fourier series coefficient

$b_{n} \quad$ Fourier series coefficient

$W_{c}(b, a)$ Continuous Wavelet Transform function

$\psi(t) \quad$ Mother wavelet

$\psi^{*}(t) \quad$ Complex conjugate of the mother wavelet

$W_{d}(t) \quad$ Continuous Wavelet Transform function

$\phi(t) \quad$ Scaling function

\section{References}

[1] J. Kubicek, M. Penhaker, and R. Kahankova, "Design of a Synthetic ECG Signal Based on the Fourier Series," p. 6.

[2] S. Bhowmick, P. K. Kundu, and G. Sarkar, "Synthesis of ECG waveform using Simulink model," in 2016 International Conference on Intelligent Control Power and Instrumentation (ICICPI), Kolkata, India, Oct. 2016, pp. 61-64. doi: 10.1109/ICICPI.2016.7859674.

[3] D. M. A. Bennet, Bhavani, and H. Priya, "Design and Analysis of Electrocardiograph (ECG) Signal for long term continuous heart rate monitoring system," p. 12. 
[4] T. Lukáč and O. Ondráček, "USING SIMULINK AND MATLAB FOR REAL-TIME ECG SIGNAL PROCESSING,”p. 6.

[5] J. Parak and J. Havlik, "ECG SIGNAL PROCESSING AND HEART RATE FREQUENCY DETECTION METHODS," p. 8.

[6] K. Murugan and R. Ramesh, "Electrocardiogram signal analysis using zoom FFT," in 2012 ISSNIP Biosignals and Biorobotics Conference: Biosignals and Robotics for Better and Safer Living (BRC), Manaus, Brazil, Jan. 2012, pp. 1-4. doi: 10.1109/BRC.2012.6222165.

[7] M. K. Islam, A. N. M. M. Haque, G. Tangim, T Ahammad, and M. R. H. Khondokar, "Study and Analysis of ECG Signal Using MATLAB \&LABVIEW as Effective Tools," IJCEE, pp. 404-408, 2012, doi: 10.7763/IJCEE.2012.V4.522.

[8] A. K. M. F. Haque, Md. H. Ali, M. A. Kiber, and Md. T. Hasan, "Detection of small variations of ECG features using wavelet," vol. 4, no. 6, p. 4, 2009.

[9] Y. Tan and L. Du, "Study on Wavelet Transform in the Processing for ECG Signals," in 2009 WRI World Congress on Software Engineering, Xiamen, China, 2009, pp. 515-518. doi: 10.1109/WCSE.2009.89.

[10] A. M. Ciupe and N. M. Roman, "Study of ECG signal processing using wavelet transforms," in 2015 9th International Symposium on Advanced Topics in Electrical Engineering (ATEE), Bucharest, Romania, May 2015, pp. 27-30. doi 10.1109/ATEE.2015.7133671

[11] Y. Talatov and T. Mgrupov, "Algorithmic and Software Analysis and Processing of ECG Signals," in 2019 International Multi-Conference on Engineering, Computer and Information Sciences (SIBIRCON), Novosibirsk, Russia, Oct. 2019, pp. 0403-0406. doi: 10.1109/SIBIRCON48586.2019.8958424.

[12] P. Sasikala and Dr. R.S.D., "Robust R Peak and QRS detection in Electrocardiogram using Wavelet Transform," IJACSA, vol. 1, no. 6, 2010, doi: 10.14569/IJACSA.2010.010608.

[13] C. Saritha, V. Sukanya, and Y. N. Murthy, "ECG Signal Analysis Using Wavelet Transforms," p. 10

[14] A. A. John, A. P. Subramanian, S. K. Jaganathan, and B. Sethuraman, "Evaluation of cardiac signals using discrete wavelet transform with MATLAB graphical user interface," Indian Heart Journal, vol. 67, no. 6, pp. 549551, Nov. 2015, doi: 10.1016/j.ihj.2015.07.017.

[15] D. Zhang et al., "An ECG Signal De-Noising Approach Based on Wavelet Energy and Sub-Band Smoothing Filter," Applied Sciences, vol. 9, no. 22, p. 4968, Nov. 2019, doi: 10.3390/app9224968.

[16] K. V. L. Narayana and A. B. Rao, "Wavelet based QRS detection in ECG using MATLAB," vol. 2, no. 7, p. 11, 2011.

[17] von Wagner, Kunzmann, Schochlin, and Bolz, "Simulation Methods for the Online Extraction of ECG Parameters under MATLAB/SIMULINK," Biosignale, vol. 47, no. 1, pp. 534-537, 2002.

[18] Liton Devnath, Subroto Kumer, D. Nath, Anup Kr, Das, and Md Rafiqul Islam, "Selection of Wavelet and Thresholding Rule for Denoising the ECG Signals," 2015, doi: 10.13140/RG.2.1.1137.6243.

[19] S. Z. Mahmoodabadi, A. Ahmadian, M. D. Abolhasani, M. Eslami, and J. H. Bidgoli, "ECG Feature Extraction Based on Multiresolution Wavelet Transform," p. 4.

[20] D. D. Patil and R. P. Singh, "ECG Classification Using Wavelet Transform and Wavelet Network Classifier," in Artificial Intelligence and Evolutionary Computations in
Engineering Systems, vol. 668, S. S. Dash, P. C. B. Naidu, R. Bayindir, and S. Das, Eds. Singapore: Springer Singapore, 2018, pp. 289-303. doi: 10.1007/978-981-107868-2_29.

[21] Z. Peng and G. Wang, "A Novel ECG Eigenvalue Detection Algorithm Based on Wavelet Transform," BioMed Research International, vol. 2017, pp. 1-12, 2017, doi: 10.1155/2017/5168346.

[22] R. J. Martis, "ECG beat classification using PCA, LDA, ICA and Discrete Wavelet Transform," Biomedical Signal Processing and Control, p. 12, 2013.

[23] A. Daamouche, L. Hamami, N. Alajlan, and F. Melgani, "A wavelet optimization approach for ECG signal classification," Biomedical Signal Processing and Control, vol. 7, no. 4, pp. 342-349, Jul. 2012, doi: 10.1016/j.bspc.2011.07.001

[24] J. A. Crowe, N. M. Gibson, M. S. Woolfson, and M. G. Somekh, "Wavelet transform as a potential tool for ECG analysis and compression," Journal of Biomedical Engineering, vol. 14, no. 3, pp. 268-272, May 1992, doi: 10.1016/0141-5425(92)90063-Q.

[25] D. T. Ingole, K. Kulat, and M. D. Ingole, "Feature Extraction via Multiresolution Analysis for ECG Signal," p. 6.

[26] Z. D. Yuan, J. Q. Xu, and G. P. Li, "Recognition of cardiac patterns based on wavelet analysis," in Proceedings of the 2003 IEEE International Symposium on Intelligent Control ISIC-03, Houston, TX, USA, 2003, pp. 642-645. doi: 10.1109/ISIC.2003.1254712.

[27] H.-Y. Lin, S.-Y. Liang, Y.-L. Ho, Y.-H. Lin, and H.-P. $\mathrm{Ma}$, "Discrete-wavelet-transform-based noise removal and feature extraction for ECG signals," IRBM, vol. 35, no. 6, pp. 351-361, Dec. 2014, doi: 10.1016/j.irbm.2014.10.004.

[28] A. Appathurai, "A study on ECG signal characterization and practical implementation of some ECG characterization techniques," p. 13, 2019.

[29] M. Adam, "Automated characterization of cardiovascular diseases using relative wavelet nonlinear features extracted from ECG signals," Computer Methods and Programs in Biomedicine, p. 11, 2018.

[30] A. Balachandran, M. Ganesan, and E. P. Sumesh, "Daubechies algorithm for highly accurate ECG feature extraction," in 2014 International Conference on Green Computing Communication and Electrical Engineering (ICGCCEE), Coimbatore, India, Mar. 2014, pp. 1-5. doi: 10.1109/ICGCCEE.2014.6922266.

[31] B. Castro, D. Kogan, and A. B. Geva, "ECG feature extraction using optimal mother wavelet," in 21st IEEE Convention of the Electrical and Electronic Engineers in Israel. Proceedings (Cat. No.00EX377), Tel-Aviv, Israel, 2000, pp. 346-350. doi: 10.1109/EEEI.2000.924422.

[32] E. M. Tamil, N. H. Kamarudin, R. Salleh, M. Y. I. Idris, M. Noor, and A. M. Tamil, "Heartbeat Electrocardiogram (ECG) Signal Feature Extraction Using Discrete Wavelet Transforms (DWT)," p. 6.

[33] S. Banerjee and M. Mitra, "ECG feature extraction and classification of anteroseptal myocardial infarction and normal subjects using discrete wavelet transform," International Conference on Systems in Medicine and Biology, p. 6, 2010.

[34] G. F. Takla and B. G. Nair, "Matching a Wavelet to ECG Signal," p. 4.

[35] S. Banerjee, "Application of Cross Wavelet Transform for ECG Pattern Analysis and Classification," IEEE TRANSACTIONS ON INSTRUMENTATION AND MEASUREMENT, vol. 63, no. 2, p. 8, 2014. 
[36] S. Banerjee and M. Mitra, "A cross wavelet transform based approach for ECG feature extraction and classification without denoising," in Proceedings of The 2014 International Conference on Control, Instrumentation, Energy and Communication (CIEC), Calcutta, India, Jan. 2014, pp. 162-165. doi: 10.1109/CIEC.2014.6959070.

[37] S. Banerjee and M. Mitra, "An approach for ECG based cardiac abnormality detection through the scope of Cross Wavelet Transform," in 2012 4th International Conference on Intelligent Human Computer Interaction (IHCI), Kharagpur, India, Dec. 2012, pp. 1-6. doi: 10.1109/IHCI.2012.6481839.

[38] S. Banerjee and M. Mitra, "Application of crosswavelet transform and Wavelet Coherence for classification of ECG patterns," in 2012 Third International Conference on Emerging Applications of Information Technology, Kolkata, West Bengal, India, Nov. 2012, pp. 89-92. doi: 10.1109/EAIT.2012.6407869.

[39] I. Janajreh and I. Talab, "Wind Data Collection and Analyses at Masdar City for Wind Turbine Assessment," IJTEE, vol. 1, no. 1, pp. 43-50, Sep. 2010, doi: 10.5383/ijtee.01.01.007.

[40] S. S. Mahmoud and J. Jusak, "Wavelets in ECG Security Application," p. 23.

[41] M. N. Dar, M. U. Akram, A. Usman, and S. A. Khan, "ECG biometric identification for general population using multiresolution analysis of DWT based features," p. 6.

[42] A. Goldberger et al., "PhysioBank, PhysioToolkit, and PhysioNet: Components of a new research resource for complex physiologic signals." PhysioNet. [Online]. Available: https://www.physionet.org/content/mitdb/1.0.0/

[43] D. S. Baim et al., "Survival of patients with severe congestive heart failure treated with oral milrinone," Journal of the American College of Cardiology, vol. 7, no. 3, pp. 661-670, Mar. 1986, doi: 10.1016/s07351097(86)80478-8.

[44] G. B. Moody and R. G. Mark, "The impact of the MITBIH Arrhythmia Database," IEEE Engineering in Medicine and Biology Magazine, vol. 20, no. 3, pp. 4550, Jun. 2001, doi: 10.1109/51.932724.

[45] S.-J. S. Tsai, "Power Transformer Partial Discharge (PD) Acoustic Signal Detection using Fiber Sensors and Wavelet Analysis, Modeling, and Simulation," Masters Thesis, Virginia Polytechnic Institute and State University, Blacksburg, Virginia, USA, 2002. [Online]. Available: http://hdl.handle.net/10919/35983

[46] C. Lowrie and S. Earles, "Wavelet Processing of Palladium Foil Images from Atomic Force Microscopy (AFM) Measurements," ECS Trans., vol. 11, no. 11, pp. 1-7, Dec. 2019, doi: 10.1149/1.2889402. 